\title{
ANÁLISIS PALINOFACIAL APLICADO A CORTEJOS SEDIMENTARIOS DEL JURÁSICO MEDIO EN EL SECTOR SUROCCIDENTAL DE LA CUENCA NEUQUINA, ARGENTINA
}

\author{
Marcelo Adrián MARTÍNEZ, Vivian Mabel GARCÍA y \\ Mirta Elena QUATTROCCHIO
}

CONICET. Universidad Nacional del Sur - Depto. de Geología. San Juan 670. (8000) Bahía Blanca. Argentina.

Martínez, M.A., García, V.M. y Quattrocchio, M.E. 1999. Análisis palinofacial aplicado a cortejos sedimentarios del Jurásico Medio en el sector suroccidental de la Cuenca Neuquina, Argentina. [Palynofacies analysis applied to systems tract from the Middle Jurassic of the southwestern Neuquén Basin, Argentina]. Revista Española de Paleontología, 14(2), 217-230. ISSN 0213-6937.

\begin{abstract}
Palynofacies analysis was used to characterize the depositional sequences JC4.1 (uppermost Toarcian lowermost Bajocian), JC4.2, JC4.3 and JC4.4 (middle Lower Bajocian), JC4.5 (middle Lower Bajocian uppermost Lower Bajocian) and JC5 (uppermost Lower Bajocian - uppermost Upper Bajocian), recognized by Zavala (1993) in the Los Molles and Lajas formations, Lohan Mahuida section, southwestern Neuquén Basin. Highstand Systems Tract (HST), Transgressive Systems Tract (TST) and Lowstand Systems Tract (LST) are recognized in each sequence. The studied palynoflora corresponds to the Callialasporites dampieri Zone (Late Toarcian - Oxfordian), Callialasporites turbatus Subzone (Late Toarcian - Early Bajocian) and Ischyosporites marburgensis Subzone (latest Early Bajocian - Late Bajocian), defined by Quattrocchio et al. (1996a). The HST are characterized by high relative abundances of Cheirolepidiaceae, Araucariaceae and spores. The marine palaeomicroplankton has the highest diversity and the phytoclasts are dominant over the amorphous organic matter. In the TST the Cheirolepidiaceae are associated with saccate pollen of Podocarpaceae and Caytoniaceae. Relative abundances of marine palaeomicroplankton are lower than in HST and the amorphous organic matter has its highest abundance. The LST are characterized by Cheirolepidiaceae, Araucariaceae and spores; the highest diversity and abundance of acritarchs, and translucent phytoclasts dominance.
\end{abstract}

Keywords: Palynofacies, systems tract, Middle Jurassic, Lohan Mahuida, Neuquén Basin, Argentina.

\section{RESUMEN}

El análisis palinofacial permitió caracterizar las secuencias deposicionales JC4.1 (Toarciense superior alto Bajociense inferior bajo), JC4.2, JC4.3 y JC4.4 (Bajociense inferior medio), JC4.5 (Bajociense inferior medio Bajociense inferior alto) y JC5 (Bajociense inferior alto - Bajociense superior alto), reconocidas por Zavala (1993) para las formaciones Los Molles y Lajas, en el perfil Lohan Mahuida del sector suroccidental de la Cuenca Neuquina, Argentina. En cada secuencia se reconocieron cortejos sedimentarios de alto nivel (HST), transgresivos (TST) y de bajo nivel (LST). La palinoflora estudiada corresponde a la Zona de Callialasporites dampieri (Toarciense tardío - Oxfordiense), Subzona de Callialasporites turbatus (Toarciense tardío - Bajociense temprano) y Subzona de Ischyosporites marburgensis (Bajociense temprano alto - Bajociense tardío), definidas por Quattrocchio et al. (1996a). Los HST se caracterizan por presentar elevados porcentajes de Cheirolepidiaceae, Araucariaceae y esporas. El paleomicroplancton marino muestra la mayor diversidad. Los fitoclastos predominan sobre la materia orgánica amorfa. En los TST las Cheirolepidiaceae están asociadas con granos de polen sacado de Podocarpaceae y Caytoniaceae. Las frecuencias relativas del paleomicroplancton marino son menores que en los HST. La materia orgánica amorfa se presenta en elevadas proporciones. Los LST se caracterizan por presentar Cheirolepidiaceae, Araucariaceae y esporas; altos valores de diversidad y frecuencias relativas de acritarcos y dominancia de fitoclastos translúcidos.

Palabras clave: Palinofacies, cortejos sedimentarios, Jurásico Medio, Lohan Mahuida, Cuenca Neuquina, Argentina. 


\section{INTRODUCCIÓN}

El presente trabajo tiene por finalidad caracterizar mediante el análisis palinofacial las secuencias deposicionales (cortejos sedimentarios $=$ systems tracts) reconocidas por Zavala (1993), en las formaciones Los Molles y Lajas en el perfil Lohan Mahuida, ubicado en el sector suroccidental de la Cuenca Neuquina.

$\mathrm{El}$ análisis palinofacial fue llevado a cabo siguiendo la clasificación de la materia orgánica palinológica propuesta por Tyson (1995). Para la determinación del tipo de materia orgánica amorfa se incorporaron modificaciones según Batten (1981) y Batten y Morrison (1983).

El presente estudio comprendió:

- la caracterización de las variaciones cuantitativas (frecuencias relativas y absolutas) de los tipos de materia palinológica presentes en las distintas secuencias deposicionales.

- la determinación de los taxones de palinomorfos presentes en cada secuencia deposicional.

- la comparación de los taxones fósiles con los actuales relacionados (Anexo) para determinar las paleocomunidades presentes en cada secuencia deposicional y sus fluctuaciones a través del tiempo.

- la determinación del grado de conservación de los palinomorfos según el reconocimiento de distintos tipos de alteración.

- la caracterización de posiciones proximales-distales con respecto al área de aporte, teniendo en cuenta las variaciones porcentuales y absolutas de los componentes analizados.

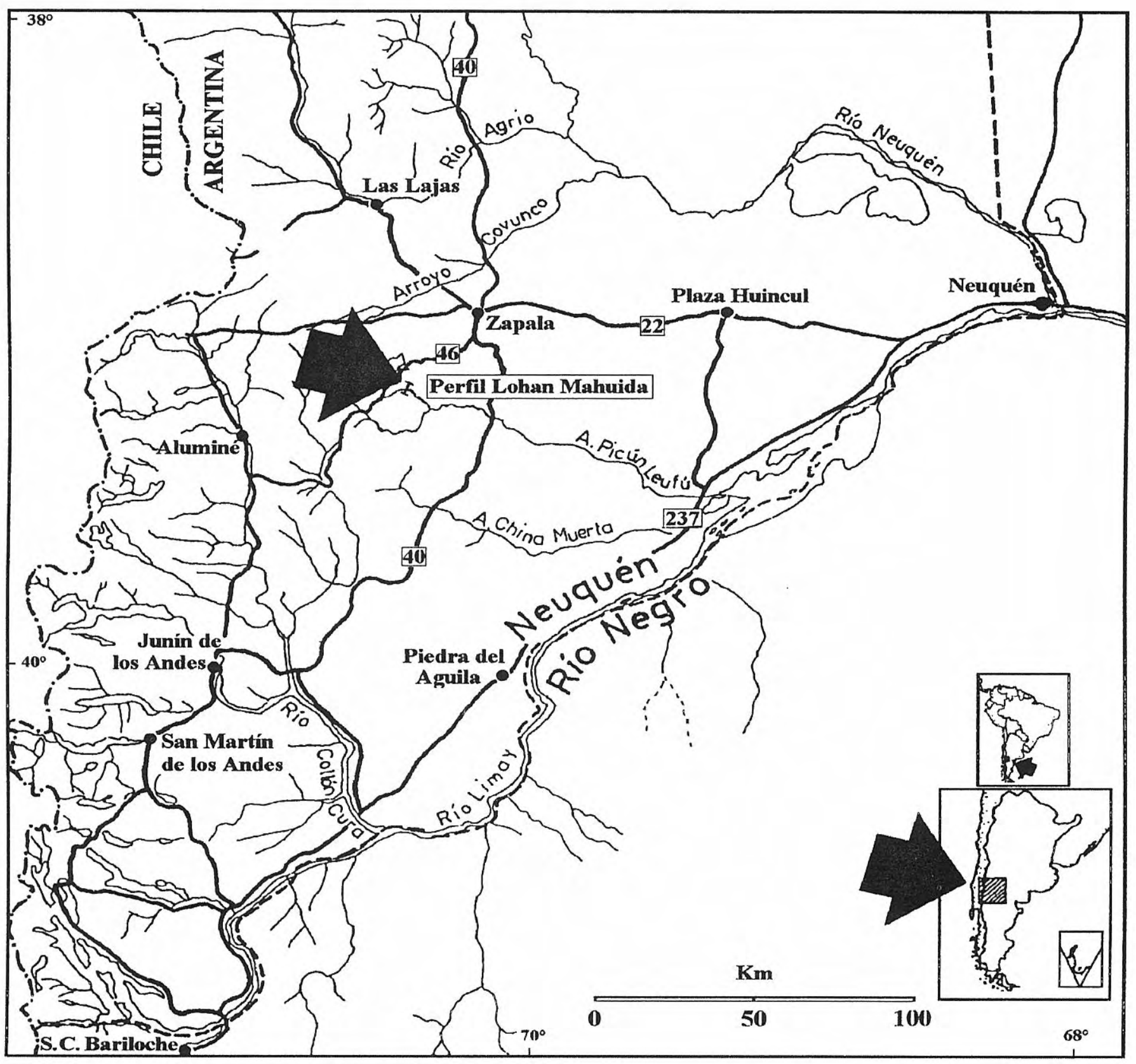

Figura 1. Mapa de ubicación del perfil Lohan Mahuida. 


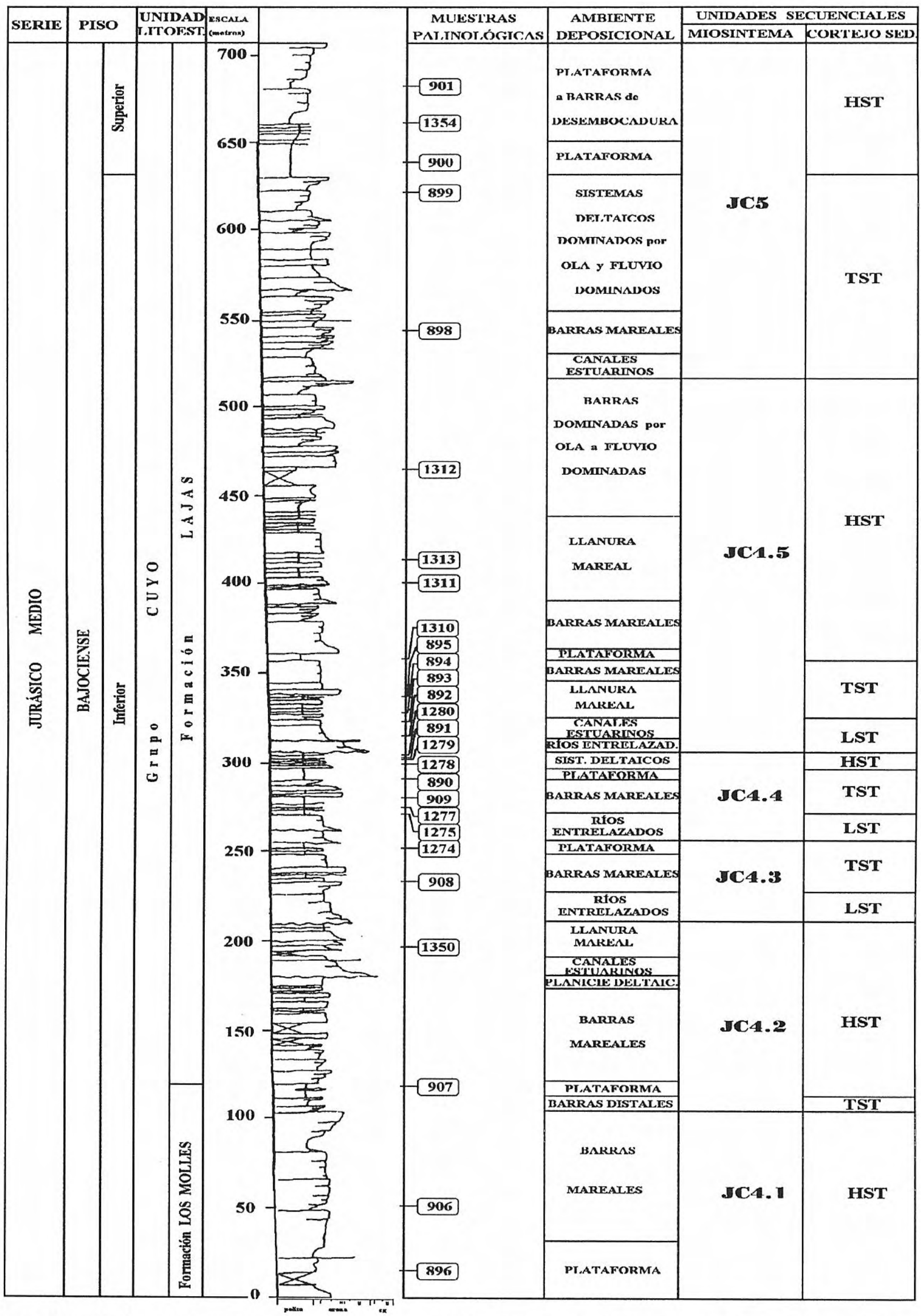

Figura 2. Perfil Lohan Mahuida (modificado de Zavala, 1993). Estratigrafía y distribución de las muestras palinológicas estudiadas. 
- a partir de la determinación de los palinomorfos se ubicó palinoestratigráficamente la sección analizada en la zonación palinológica realizada para la Cuenca Neuquina por Quattrocchio et al. (1996a).

\section{UBICACIÓN DEL ÁREA, ESTRATIGRAFIA, EDAD Y ANTECEDENTES}

La Cuenca Neuquina es una cuenca de retroarco, localizada en el sector centro-occidental de Argentina. Se originó durante el Triásico tardío y constituyó una importante región de sedimentación durante el Jurásico y Cretácico temprano. El Grupo Cuyo (Jurásico temprano a medio), representa el primer episodio de deposición después de la configuración de la cuenca. El área de estudio se localiza en el sector suroccidental de la Cuenca Neuquina, Argentina, a los $39^{\circ} 10^{\prime}$ de latitud sur y $70^{\circ}$ $25^{\prime}$ de longitud oeste (Fig. 1). El análisis palinofacial fue aplicado a muestras provenientes del Perfil Lohan Mahuida (Fig. 2). Los depósitos corresponden al Bajociense inferior a Bajociense superior, formaciones Los Molles y Lajas, datados por fauna de ammonites (Zavala, 1993), Zona de Asociación de Emileia giebeli (Westermann y Riccardi, 1979), correspondiente al Bajociense temprano y la Zona de Asociación de Megasphaeroceras rotundum (Hall y Westermann, 1980), correspondiente al Bajociense tardío.

El perfil Lohan Mahuida de 708 metros de espesor comprende las secuencias deposicionales JC4.1 (Toarciense superior alto - Bajociense inferior bajo), JC4.2, JC4.3 y JC4.4 (Bajociense inferior medio), JC4.5 (Bajociense inferior medio - Bajociense inferior alto) y JC5 (Bajociense inferior alto - Bajociense superior alto), (Zavala, 1993, 1996a y 1996b). A estas secuencias deposicionales se las considera internamente divididas en unidades estratigráficas más pequeñas que constituyen una cadena de sistemas deposicionales contemporáneos, denominados cortejos sedimentarios (System Tracts). Estos se clasifican en cortejos sedimentarios de alto nivel (HST), cortejos sedimentarios transgresivos (TST) y cortejos sedimentarios de bajo nivel (LST). En el área de estudio, Zavala, (1993) reconoce para los distintos cortejos sedimentarios los siguientes ambientes deposicionales: dentro de los HST ambientes de plataforma - barras de desembocadura - llanura mareal barras dominadas por ola y fluvio dominadas - sistemas deltaicos; para los TST reconoce barras mareales sistemas deltaicos dominados por ola - llanura mareal plataforma y para los LST canales estuarinos.

Existen escasos antecedentes de estudios palinofaciales realizados en Argentina. En García et al. (1994), se muestra la relación entre el análisis palinológico y el análisis de facies aplicado a depósitos del Grupo Cuyo, en la Sierra de Chacaico, Cuenca Neuquina. Las primeras menciones de estudios palinofaciales en la Cuenca Neuquina corresponden a Quattrocchio et al. (1996b).

\section{METODOLOGÍA}

La extracción físico-química de las muestras palinológicas (27 niveles) se efectuó sometiendo el material al ataque con ácido clorhídrico (10\%) en frío para disolver carbonatos, y ácido fluorhídrico concentrado (70\%) en frío durante 48 horas para disolver los silicatos. A partir de este residuo se realizaron preparados para estudiar el contenido orgánico total. Batten (1981) y Batten y Morrison (1983) aconsejan un breve tratamiento del residuo orgánico (2 minutos) con oxidación y/o ultrasonido, en los casos en que la materia orgánica amorfa presente dudas acerca de su origen, ya sea algal o derivado de plantas vasculares. El material amorfo derivado de plantas vasculares es más fácilmente removido que el de origen algal tras la oxidación y/o ultrasonido. En algunos casos el estudio de las

\section{Lámina I}

1 Antulsporites saevus (Balme) Archangelsky y Gamerro 1966. Muestra 1350 b: 11,5/126,5. Vista proximal, $x$ 1000. Diámetro ecuatorial máximo: $33 \mu \mathrm{m}$.

2 Todisporites minor Couper 1958. Muestra 908 b: 24,3/132,3. Vista proximal, x 1000. Diámetro ecuatorial máximo: $44 \mu \mathrm{m}$.

3 Ischyosporites marburgensis De Jersey 1963. Muestra 895 c: 19,1/140,7. Vista distal, x 1000. Diámetro ecuatorial máximo: $39 \mu \mathrm{m}$.

4 Verrucosisporites varians Volkheimer 1972. Muestra 891 b: 6,5/129. Vista proximal, x 1000. Diámetro ecuatorial máximo: $40 \mu \mathrm{m}$.

5 Callialasporites segmentatus (Balme) Srivastava 1963. Muestra 901 c: 7/147. Vista polar, x 1000. Diámetro ecuatorial máximo: $62 \mu \mathrm{m}$.

6 Callialasporites dampieri (Balme) Dev 1961. Muestra 907 c: 14,4/119,8. Vista polar, x 1000. Diámetro ecuatorial máximo: $50 \mu \mathrm{m}$.
7 Vitreisporites pallidus (Reissinger) Nilsson 1958. Muestra 890 d: 23/139,5. Vista polar, x 1000. Largo total: $23 \mu \mathrm{m}$.

8 Podocarpidites spp. Muestra $908 \mathrm{~d}:$ 12,5/148. Vista lateral, x 1000. Largo total: $35 \mu \mathrm{m}$.

9 Inaperturopollenites turbatus Balme 1957. Muestra 900 e: 15,4/134, x 1000. Diámetro máximo: $82 \mu \mathrm{m}$.

10 Classopollis cf. classoides (Pflug) Pocock y Jansonius (en Volkheimer, 1968). Muestra 893 a: 3,3/139,5. Vista oblicua, x 1000. Diámetro ecuatorial máximo: $33 \mu \mathrm{m}$.

11 Nannoceratopsis gracilis (Alberti 1961) Evitt 1962. Muestra 891b: 5,5/142,6, x 1000. Largo total: $66 \mu \mathrm{m}$.

12 Michrystridium fragile Deflandre 1947b. Muestra $901 \mathrm{~d}$ : 23,3/138, x 1000. Diámetro máximo de la vesícula: 17 $\mu \mathrm{m}$.

13 Escharisphaeridia pocockii (Sarjeant 1968) Erkmen y Sarjeant 1980. Muestra 908 b: 13,2/144, x 1000. Largo total (sin opérculo): $40 \mu \mathrm{m}$. 
Lámina I

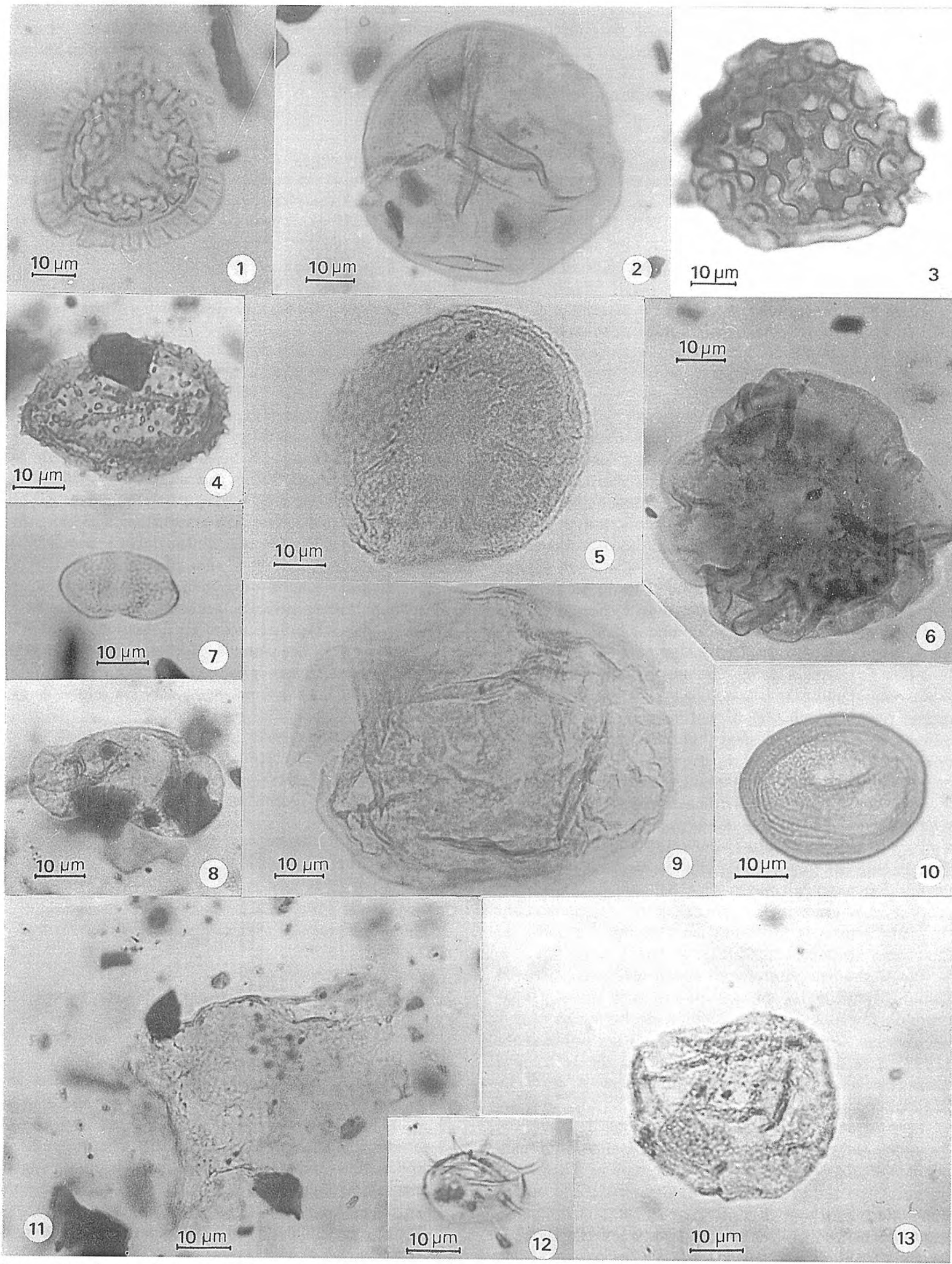

Revista Española de Paleontología, 14 (2), 1999. 
asociaciones microflorísticas requirió mayores tiempos de oxidación (ácido nítrico), remoción de compuestos húmicos (hidróxido de amonio), filtrado y/o tratamiento con líquidos pesados (cloruro de cinc). Los preparados definitivos se montaron en glicerina gelatina y el residuo palinológico fue preservado agregando solución de formol. Las muestras están archivadas en el Laboratorio de Palinología de la Universidad Nacional del Sur.

El estudio de las muestras se realizó mediante técnicas de microscopía de luz transmitida, utilizando un microscopio Olympus BH $2 \mathrm{~N}^{\circ}$ 100786. Las microfotografías fueron obtenidas con una cámara Olympus automática y se utilizó película Kodak de 100 ASA (Lám. I).

El análisis palinofacial (estudio de la materia orgánica total contenida en un residuo palinológico) se realizó con base en cuatro tipos de materia orgánica según Tyson (1995): palinomorfos, fitoclastos (translúcidos y opacos), tipo amorfo y zooclastos (Fig. 3).

Los palinomorfos se clasificaron en 10 grupos supragenéricos: esporas, Caytoniaceae, Podocarpaceae, Araucariaceae, Cheirolepidiaceae, Cycadales/Bennettitales, Chlorococcales, acritarcos, dinoquistes y Prasinophyceae (Anexo). El análisis estadístico de las asociaciones microflorísticas se realizó a partir de recuentos polínicos (200 palinomorfos), obteniéndose valores de frecuencias relativas para cada grupo taxonómico. A partir del agregado de polen foráneo (tabletas conteniendo 11267 esporas de Lycopodium sp.) se obtuvieron las frecuencias absolutas o concentraciones totales para cada muestra (granos/gramos de sedimento). El orden de mención de los distintos componentes de las asociaciones microflorísticas, corresponde a su mayor o menor representación en la palinofacies analizada. El número de especies terrestres y marinas se utilizó para la construcción de curvas de diversidad (números de especies por muestra). El contenido de fitoclastos, amorfo y zooclastos se determinó mediante valoraciones visuales realizadas en cinco puntos del preparado con un objetivo x 40; y se expresó como frecuencias relativas independientemente del contenido de palinomorfos, según Batten (1987). El estado de conservación del contenido orgánico (corrosión, degradación, daño mecánico y cristalización de pirita dentro de la exina), se determinó a partir de Delcourt y Delcourt (1980) (Fig. 4).

Las diferentes palinofacies identificadas en el Perfil Lohan Mahuida fueron agrupadas para los distintos cortejos sedimentarios en función de los ambientes y secuencias deposicionales identificadas por Zavala (1993). Las mismas fueron designadas desde la base hacia el techo en el perfil como palinofacies A, B, C, D, E, F, G, H, I y J, (Fig. 3).

Para determinar las tendencias proximal/distal al área de aporte para cada nivel, se utilizaron los siguientes criterios según Tyson (1995):

\section{Proximal}

predomino de fitoclastos translúcidos alta relación fitoclastos/amorfo esporas $>$ polen alta relación esporas/bisacados baja diversidad de paleomicroplancton

baja relación esporas/bisacados

Prasinophyceae $\geq$ dinoquistes

alta diversidad de paleomicroplancton dinoquistes $>$ Prasinophyceae

\section{RESULTADOS}

Del análisis palinofacial surgen 10 palinofacies, caracterizándose las mismas en función de la asociación microflorística característica, proporción de fitoclastos y amorfo, clases de alteración y diversidad de especies terrestres y marinas:

\section{Palinofacies A HST - JC4.1}

Facies de plataforma a barras mareales. Muestras 896 y 906.

- Asociación característica

Terrestre: Cheirolepidiaceae - Araucariaceae y/o esporas.

Marino: Baltisphaeridium debilispinum - Micrhystridium fragile - Cymatiosphaera eupeplos - Leiosphaeridia sp. C Escharisphaeridia pocockii.

En esta asociación se registra el predominio de las Cheirolepidiaceae con frecuencias absolutas y relativas que varían entre $2742(68,1 \%)$ y $4791(91,27 \%)$ granos. $\mathrm{g}^{-1}$. Las Araucariaceae representan valores entre $122(2,38 \%)$ y 244 $(5,99 \%)$ granos.g $\mathrm{g}^{-1}$, las esporas $150(3,69 \%)$ y $203(3,97 \%)$ granos $\cdot \mathrm{g}^{-1}$.

El paleomicroplancton marino sólo se ha registrado en depósitos de barras mareales. Los acritarcos representan el segundo grupo supragenérico en abundancia con 470 $(11,6 \%)$ granos. $\mathrm{g}^{-1}$. Los dinoquistes representan $188(4,61 \%)$ granos. $\mathrm{g}^{-1}$, con Escharisphaeridia pocockii como única especie hallada. Las Prasinophyceae alcanzan valores de 112 $(2,76 \%)$ granos $\cdot \mathrm{g}^{-1}$.

- Otros constituyentes orgánicos: amorfo (40-50\%), fitoclastos translúcidos (30\%) y fitoclastos opacos (20-30\%).

- Clases de alteración: son predominantes el daño mecánico, cristalización de pirita dentro de la exina y degradación.

- Diversidad: terrestre: 5 - 10 especies, marino: 0 - 6 especies.

\section{Palinofacies B HST - JC4.2}

Facies de prodelta a llanura mareal. Muestras 907 y 1350.

- Asociación característica

Terrestre: Cheirolepidiaceae - Araucariaceae y/o Podocarpaceae.

Marino: Escharisphaeridia pocockii - Acanthaulax spp. Cymatiosphaera sp. - Micrhystridium sp.

Las Cheirolepidiaceae presentan las mayores frecuencias con valores de 4421 granos $\cdot \mathrm{g}^{-1},(46,53 \%)$ a 23968 granos $\cdot \mathrm{g}^{-1}$ $(62,9 \%)$. Las Araucariaceae presentan valores entre 3687 $(9,68 \%)$ y $2438(25,98 \%)$ granos.g ${ }^{-1}$, constituyendo el segundo grupo supragenérico en importancia en facies correspondientes a prodelta. Las Podocarpaceae varían entre $1219(13 \%)$ y $6146(16,1 \%)$ granos. $\mathrm{g}^{-1}$.

El paleomicroplancton marino presenta los siguientes valores: los dinoquistes varían entre $227(2,42 \%)$ y 410 $(1,08 \%)$ granos. $\mathrm{g}^{-1} ;$ los acritarcos, entre $205(0,54 \%)$ y 146 
Muestras (Palinofacies)

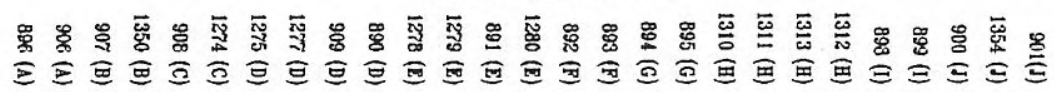

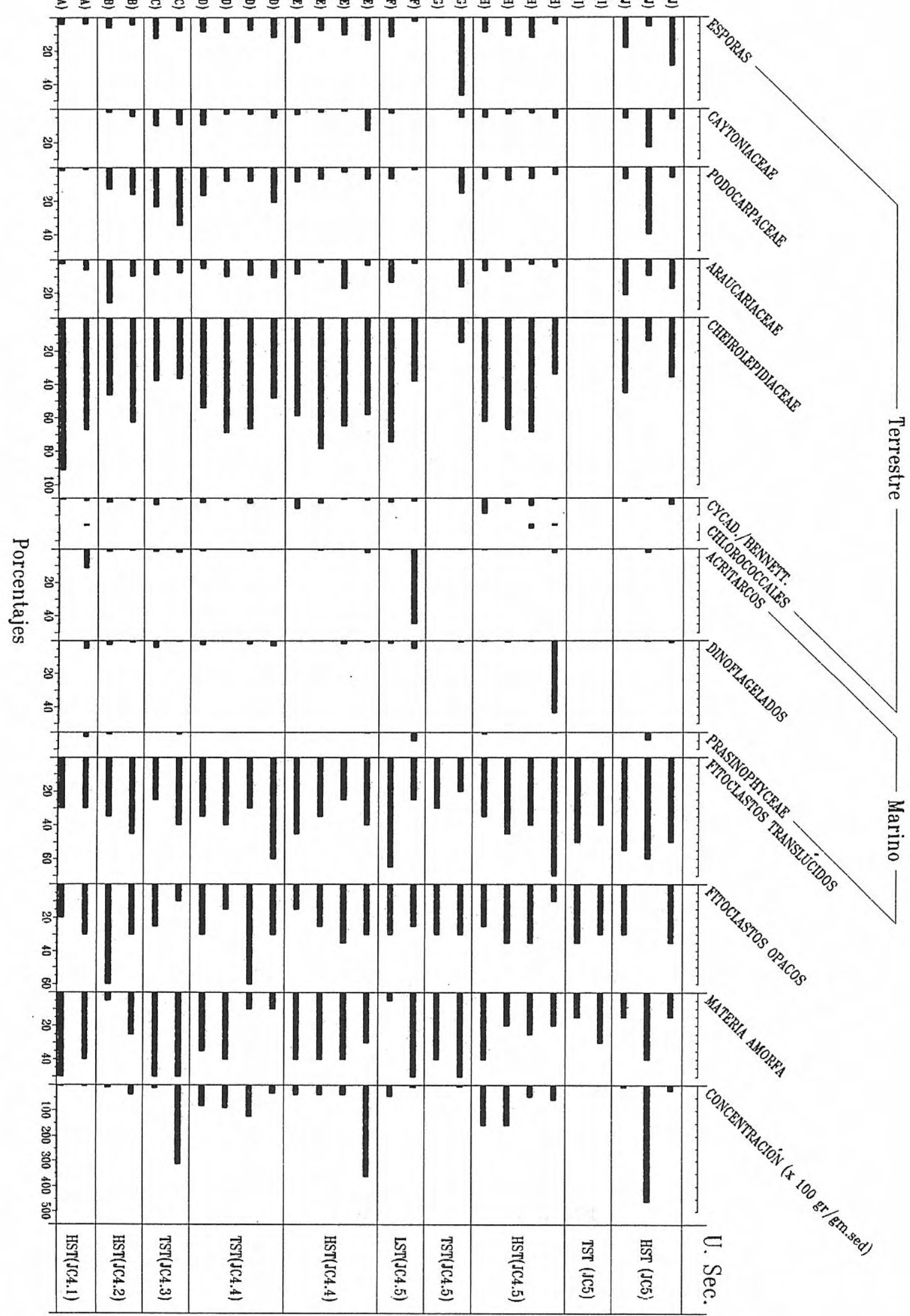

Figura 3. Representación de las palinofacies según las proporciones de palinomorfos, fitoclastos y materia orgánica amorfa. Concentración total de cada palinofacies. 
$(1,81 \%)$ granos $\cdot \mathrm{g}^{-1}$; las Prasinophyceae se presentan en facies de prodelta con $24(0,91 \%)$ granos. $\mathrm{g}^{-1}$.

Los valores más elevados de frecuencias absolutas de los grupos supragenéricos corresponden a las facies de llanura mareal.

- Otros constituyentes orgánicos: fitoclastos opacos (30$60 \%$ ), fitoclastos translúcidos (35-45\%), amorfo (5-25\%).

- Clases de alteración: son predominantes el daño mecánico, cristalización de pirita dentro de la exina. La degradación se ha observado en facies de llanura mareal.

- Diversidad: terrestre: 16 - 20 especies, marino: 2 - 3 especies.

\section{Palinofacies C TST - JC4.3}

Facies de barras mareales a plataforma. Muestras 908 y 1274.

- Asociación característica

Terrestre: Cheirolepidiaceae - Podocarpaceae - esporas y/o Caytoniaceae.

Marino: Escharisphaeridia pocockii - Pleurozonaria picunensis - Baltisphaeridium sp. - Micrhystridium fragile.

Las Cheirolepidiaceae se presentan con valores que varían entre $4457(37,87 \%)$ y $114172(36,54 \%)$ granos.g ${ }^{-1}$. El segundo grupo supragenérico en importancia corresponde a las Podocarpaceae cuyos valores oscilan entre 2754 $(23,4 \%)$ y $108163(34,62 \%)$ granos $\cdot g^{-1}$. Las esporas presentan valores entre $1252(12,34 \%)$ y $24036(7,69 \%)$ granos. $\mathrm{g}^{-1}$; en tanto que las Caytoniaceae oscilan entre 11152 $(9,79 \%)$ y $28543(9,13 \%)$ granos. $\mathrm{g}^{-1}$; y las Araucariaceae entre $1052(8,94 \%)$ granos $\cdot \mathrm{g}^{-1}$ y $24036(7,69 \%)$ granos $\cdot \mathrm{g}^{-1}$.

Dentro del paleomicroplancton los dinoquistes presentan valores entre $501(4,26 \%)$ granos.g ${ }^{-1}$ y 1502 $(0,48 \%)$ granos $\cdot \mathrm{g}^{-1}$; los acritarcos, entre $150(1,28 \%)$ granos $\cdot \mathrm{g}^{-1}$ y $6009(1,92 \%)$ granos $\cdot \mathrm{g}^{-1}$. Las Prasinophyceae solo se presentan en facies de plataforma con frecuencias absolutas de 3005 (0,96\%) granos. $\mathrm{g}^{-1}$. Las frecuencias absolutas muestran los valores más elevados en facies de ambiente de plataforma (312471 granos. $\mathrm{g}^{-1}$ ), mientras que para facies de barras mareales se registran valores de 11768 granos $\cdot \mathrm{g}^{-1}$.

- Otros constituyentes orgánicos: amorfo (50\%), fitoclastos translúcidos entre 25 y $40 \%$ y fitoclastos opacos entre 10 a $25 \%$.

- Clases de alteración: son predominantes la degradación y el daño mecánico. En facies de plataforma la corrosión y la cristalización de pirita dentro de la exina se presentan en forma subordinada, en tanto que en facies de barras mareales la cristalización de pirita es predominante.

- Diversidad: terrestre: 15 -16 especies, marino: 3 especies.

\section{Palinofacies D TST - JC4.4}

Facies de barras mareales a plataforma. Muestras 1275, 1277, 909 y 890.

\section{- Asociación característica}

Terrestre: Cheirolepidiaceae - Podocarpaceae Araucariaceae y/o esporas.

Marino: Escharisphaeridia pocockii - Nannoceratopsis sp. - Kalyptea cf. jurassica - Micrhystridium fragile Baltisphaeridium sp.
Las Cheirolepidiaceae constituyen el grupo supragenérico más abundante, con frecuencias que oscilan entre $15345(48,5 \%)$ granos $\cdot \mathrm{g}^{-1}$ y $81874(66,73 \%)$ granos $\cdot \mathrm{g}^{-1}$. Las Podocarpaceae alcanzan frecuencias absolutas entre 6653 y 13896 granos. g $^{-1}$, y frecuencias relativas entre 8,27 y $20,9 \%$. Las Araucariaceae y esporas se presentan con frecuencias relativas del orden del $10 \%$ y en general no superan los 10000 granos.g ${ }^{-1}$.

El paleomicroplancton marino presenta valores entre $1,84 \%$ y $3,03 \%$ para los dinoquistes con frecuencias absolutas que varían entre 966 y 2253 granos.g-1. Los acritarcos no superan el $1 \%$.

- Otros constituyentes orgánicos: el contenido orgánico varía entre un amplio rango de frecuencias. Para el tramo inferior (muestras 1275 y 1277) del cortejo sedimentario transgresivo, el amorfo y los fitoclastos translúcidos alcanzan valores del orden del $40 \%$ cada uno. Para el tramo superior (muestras 909 y 890) la proporción de amorfo se reduce a un $10 \%$.

- Clases de alteración: en el tramo inferior del cortejo sedimentario transgresivo se observa un predominio de daño mecánico, corrosión y cristalización de pirita en la exina de los palinomorfos; en tanto que para el tramo superior sólo se observa en forma dominante el daño mecánico y cristalización de pirita.

- Diversidad: terrestre: 16 - 25 especies, marino: hasta 4 especies.

\section{Palinofacies E HST - JC4.4}

Facies de sistemas deltaicos. Muestras 1278, 1279, 891 y 1280 .

- Asociación característica

Terrestre: Cheirolepidiaceae - esporas .

Marino: Escharisphaeridia pocockii - Nannoceratopsis gracilis - Leiosphaeridia ef $L$. sp. B.

Las Cheirolepidiaceae representan entre el 58,09 y el $78,46 \%$ y las frecuencias absolutas varían entre 21783 y 210317 granos. $g^{-1}$. Las esporas constituyen el segundo grupo supragenérico en abundancia con frecuencias relativas que oscilan entre 7,69 y $15,15 \%$, mientras que las frecuencias absolutas varían entre 2817 y 49575 granos $\cdot \mathrm{g}^{-1}$. Las Araucariaceae, Podocarpaceae y Caytoniaceae en general adquieren valores del orden del $10 \%$.

El paleomicroplancton marino posee escasa representación, variando los dinoquistes entre $0,83 \%$ y $1,98 \%$. Los acritarcos presentan valores entre 0,51 y $2,07 \%$ correspondiendo la mayoría a la categoría de indeterminados. Las Prasinophyceae representan el 0,83\%.

- Otros constituyentes orgánicos: amorfo (30-40\%), fitoclastos translúcidos (25-45\%), fitoclastos opacos (15$40 \%)$.

- Clases de alteración: se presenta en forma predominante el daño mecánico y la cristalización de pirita dentro de la exina, manifestándose en forma subordinada los restantes tipos de alteración.

- Diversidad: terrestre: 17 - 26 especies, marino: hasta 3 especies.

Palinofacies F LST - JC4.5

Facies de canales estuarinos. Muestras 892 y 893. 


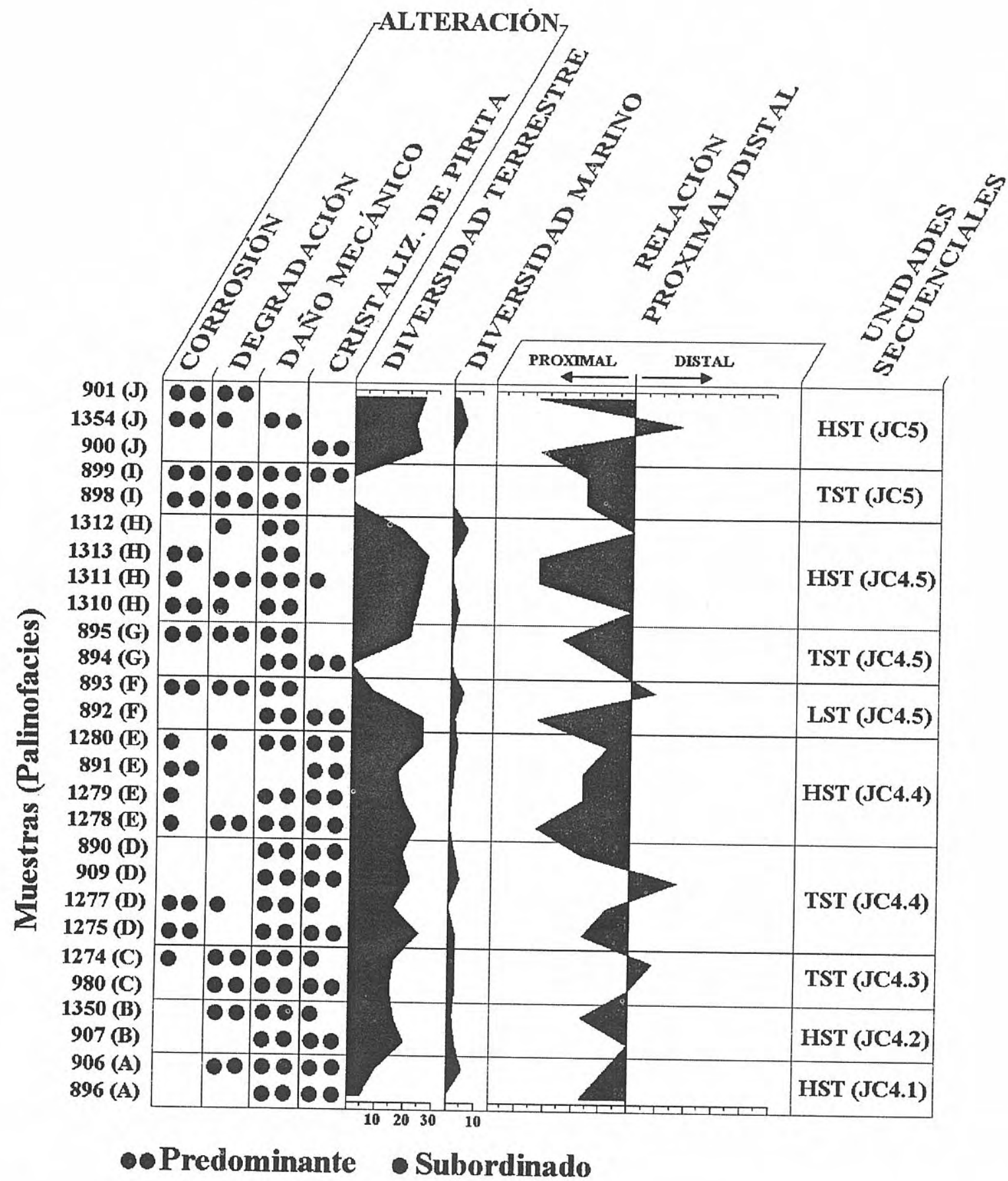

Figura 4. Caracterización del tipo de alteración, diversidad de los componentes marinos y terrestres y relación proximal/distal de cada palinofacies.

- Asociación característica

Terrestre: Cheirolepidiaceae - Araucariaceae y/o esporas. Marino: Comasphaeridium sp. - Baltisphaeridium sp. Micrhystridium fragile - Solisphaeridium sp. Cymatiosphaera eupeplos - Escharisphaeridia pocockii.

Las Cheirolepidiaceae presentan valores entre 3439 $(37,96 \%)$ y $26521(74,50 \%)$ granos. $\mathrm{g}^{-1}$. El segundo grupo supragenérico en importancia lo constituyen las Araucariaceae y esporas, con similares proporciones en sus frecuencias relativas, que varían entre $2,31 \%$ y $13,55 \%$, y absolutas entre 6000 y 120000 granos.g ${ }^{-1}$. Los restantes constituyentes terrestres poseen escasa representación.
El paleomicroplancton marino constituye el grupo supragenérico dominante en facies de llanura mareal. Los acritarcos alcanzan valores de 3798 (49,54\%) granos. $\mathrm{g}^{-1}$, las Prasinophyceae $430(5 \%)$ granos $\cdot \mathrm{g}^{-1}$ y los dinoquistes 395 $(4,63 \%)$ granos $\cdot \mathrm{g}^{-1}$. Presencia de foraminíferos.

- Otros constituyentes orgánicos: fitoclastos translúcidos (25-65\%), fitoclastos opacos (25-30\%), amorfo (5-50\%).

- Clases de alteración: en la base del cortejo sedimentario de bajo nivel se observa daño mecánico y cristalización de pirita en la exina de los palinomorfos. En facies de llanura mareal predomina el daño mecánico, la degradación y la corrosión. 
- Diversidad: terrestre: 8 - 26 especies, marino: 2 - 5 especies.

\section{Palinofacies G TST - JC4.5}

Facies de llanura mareal. Muestras 894 y 895.

- Asociación característica

Terrestre: esporas - Araucariaceae - Podocarpaceae Cheirolepidiaceae.

Marino: dinoquistes indeterminados.

Las esporas de pteridofitas representan el grupo supragenérico más abundante en esta asociación, con frecuencias absolutas del orden de $2699(46,77 \%)$ granos. $\mathrm{g}^{-1}$. Con proporciones del orden del $15 \%$ (900 granos. $\mathrm{g}^{-1}$ ) se presentan como segundo grupo supragenérico en importancia las Araucariaceae, Podocarpaceae y Cheirolepidiaceae.

El paleomicroplanton marino se halla escasamente representado, reconociéndose sólo dinoquistes indeterminados.

- Otros constituyentes orgánicos: amorfo (40-50\%), fitoclastos opacos (30\%) y fitoclastos translúcidos (20-30\%).

- Clases de alteración: en el tramo inferior (muestra estéril) se reconoce daño mecánico y cristalización de pirita dentro de la exina. Para el tramo superior predomina corrosión, degradación y daño mecánico.

- Diversidad: terrestre: 21 especies, marino: 1 especie.

\section{Palinofacies H HST - JC4.5}

Facies de plataforma, llanura mareal y barras dominadas por ola y fluvio dominadas. Muestras 1310, 1311, 1313 y 1312.

- Asociación característica

Terrestre: Cheirolepidiaceae - esporas - Podocarpaceae.

Marino: Leiosphaeridia sp. C - Micrhystridium cf. rarispinum - Endoscrinium spp. - Escharisphaeridia spp. En el nivel 1312, Jansonia sp. - Escharisphaeridia pocockii Solisphaeridium stimuliferum - Acanthaulax (?) downiei Micrhystridium spp.

Las Cheirolepidiaceae constituyen el grupo supragenérico más importante con frecuencias relativas que varían entre 62,1 y $68,57 \%$ y frecuencias absolutas entre 30904 y 105159 granos $\cdot \mathrm{g}^{-1}$. Las esporas representan entre 8,53 y $12,14 \%$ (5473 - 17276 granos. $\left.\mathrm{g}^{-1}\right)$. Las Podocarpaceae constituyen el tercer grupo en abundancia con frecuencias relativas cercanas al 7\% (3058 - 12018 granos. $\left.\mathrm{g}^{-1}\right)$. Las Cycadales/Bennettitales, de escasa representación en todo el perfil, adquieren aquí sus mayores frecuencias, 3,35 - 9\% y 1931 - 14272 granos. $\mathrm{g}^{-1}$.

El paleomicroplancton marino (dinoquistes + acritarcos + Prasinophyceae), no supera el $4 \%$. Una excepción es el nivel 1312 para el que se menciona una asociación dominada por paleomicroplancton marino (45\%, aproximadamente 26000 granos. $\mathrm{g}^{-1}$ ). Jansonia sp. (quiste de dinoflagelado) representa valores cercanos al $40 \%$, sugiriendo que fue un taxón oportunista que proliferó cuando prevalecieron condiciones ambientales inestables de agua salobre (Martínez et al., 1998). Las Cheirolepidiaceae representan el segundo grupo supragenérico, con $34 \%$ (19154 granos. $\left.\mathrm{g}^{-1}\right)$. Las esporas, Caytoniaceae, Podocarpaceae y Araucariaceae se presentan cada una con frecuencias relativas cercanas al $5 \%$.

- Otros constituyentes orgánicos: fitoclastos translúcidos (35-45\%), amorfo (20-40\%) y fitoclastos opacos (25-35\%). En el nivel 1312: fitoclastos translúcidos (70\%), amorfo: $20 \%$ y fitoclastos opacos: $10 \%$.

- Clases de alteración: el daño mecánico y la corrosión se manifiestan como los tipos de alteración más frecuentes, mientras que la degradación y por último la cristalización de pirita dentro de la exina lo hacen en forma más esporádica. En el nivel 1312, predominio de daño mecánico, menos frecuente, degradación.

- Diversidad: terrestre: 23 - 27 especies, marino: 1 - 3 especies. En el nivel 1312, terrestre: 18 y marino: 6.

\section{Palinofacies I TST - JC5}

Facies de barras mareales a sistemas deltaicos dominados por ola. Muestras 898 y 899 .

Niveles estratigráficos en los que solamente se registró la presencia de restos de Classopollis en muy mal estado de conservación.

- Otros constituyentes orgánicos: fitoclastos translúcidos (40-50\%), fitoclastos opacos (30-35\%), amorfo (10-15\%).

- Clases de alteración: se presentan en forma dominante corrosión, degradación y daño mecánico. En las facies correspondientes a sistemas deltaicos domina también la cristalización de pirita (moldes dentro de los restos de palinomorfos).

\section{Palinofacies J HST-JC5}

Facies de plataforma a barras de desembocadura. Muestras 900, 1354 y 901.

- Asociación característica

Terrestre: Cheirolepidiaceae - esporas y/o Araucariaceae.

Podocarpaceae - Caytoniaceae - Cheirolepidiaceae (en posición más distal al área de aporte).

Marino: dinoquistes indeterminados.

En posición más distal al área de aporte: Leiosphaeridia sp. C - Cymatiosphaera eupeplos - Pterosphaeridia spp.

Dominan en esta asociación las Cheirolepidiaceae (35,71-45,35\%), las frecuencias absolutas varían entre 3899 y 7511 granos.g ${ }^{-1}$. Las esporas y las Araucariaceae se presenta con similares proporciones (17-29\%), con valores de frecuencias absolutas que varían entre 1500 y 6000 granos. $\mathrm{g}^{-1}$.

El paleomicroplancton marino es escaso y pobremente conservado en facies proximales. En facies distales al área de aporte, la asociación microflorística está caracterizada por el predominio de Podocarpaceae (40,2 \%, 184779 granos. $\left.\mathrm{g}^{-1}\right)$ y Caytoniaceae $\left(23,04 \%, 105910\right.$ granos. $\left.\mathrm{g}^{-1}\right)$, mientras que las Cheirolepidiaceae alcanzan el $13,73 \%$ y valores absolutos de 63095 granos.g ${ }^{-1}$. Dentro del paleomicroplancton marino predominan las Prasinophyceae $(4,41 \%$, 20281 granos $\left.\cdot \mathrm{g}^{-1}\right)$.

- Otros constituyentes orgánicos: en facies proximales los fitoclastos translúcidos varían entre $(50-60 \%)$, los fitoclastos opacos entre (30-35\%). Amorfo $15 \%$, hasta $40 \%$ en facies distales.

- Clases de alteración: predominan corrosión y degradación y ocasionalmente se presenta daño mecánico y cristalización de pirita. 
- Diversidad: terrestre: 22 - 24 especies, marino: hasta 5 especies.

\section{CONCLUSIONES}

Debido a la presencia de palinomorfos correspondientes al "Complejo" Callialasporites $(C$. dampieri, $C$. microvelatus, C. segmentatus, C. trilobatus), Ischyosporites marburgensis, Podocarpidites verrucosus, Microcachryidites spp., y, dentro del paleomicroplancton marino, dinoquistes pertenecientes a los géneros Endoscrinium y Nannoceratopsis, las palinofloras analizadas se correlacionan con la Zona de Callialasporites dampieri (Toarciense tardío Oxfordiense), Subzona de Callialasporites turbatus (Toarciense tardío - Bajociense temprano) y la Subzona de Ischyosporites marburgensis (Bajociense temprano alto - Bajociense tardío), definidas por Quattrocchio et al. (1996a).

Según lo observado en Martínez et al. (1996), las configuraciones palinofaciales de los cortejos sedimentarios dependen de diversos factores, no sólo eustáticos, que parece ser el factor principal en este caso (Zavala, com. personal), sino también climáticos y tectónicos. Por ello, la caracterización mediante el análisis palinofacial de las secuencias deposicionales reconocidas en el perfil Lohan Mahuida permite dar las siguientes generalizaciones con respecto a los distintos cortejos sedimentarios reconocidos para el sector suroccidental de la Cuenca Neuquina y para el intervalo de tiempo geológico en estudio

Los cortejos sedimentarios de alto nivel se caracterizan por presentar:

- Elevados porcentajes de Cheirolepidiaceae, Araucariaceae y/o esporas. Las Podocarpaceae superan a las Araucariaceae y/o esporas, en las facies más distales al área de aporte. La parte superior de JC4.2 presenta estas características; pero, siguiendo a Quattrocchio et al. (1996b) y Martínez et al. (1996), podría asociarse a un relativo deterioro climático.

- El paleomicroplancton marino alcanza los mayores valores en diversidad (6 especies), siendo los dinoquistes los más abundantes (5\%).

- Las mayores frecuencias absolutas totales se hallaron asociadas a barras de desembocadura (460000 granos $\left.\cdot \mathrm{g}^{-1}\right)$.

- Los fitoclastos superan a la materia orgánica amorfa, excepto en facies más distales donde las proporciones de ambos son similares. Los fitoclastos translúcidos son más abundantes que los fitoclastos opacos.

- La alteración predominante es el daño mecánico y la cristalización de pirita.

Los cortejos sedimentarios transgresivos se caracterizan por:

- El predominio de Cheirolepidiaceae y polen sacado (Podocarpaceae y Caytoniaceae). Las esporas alcanzan hasta $50 \%$ en las facies más proximales al área de aporte (llanura mareal).

- El paleomicroplancton marino presenta baja diversidad (3 especies) y no supera el 5\%.

- Las mayores frecuencias absolutas totales se hallaron en facies de plataforma abierta con 312471 granos $\cdot g^{-1}$.

- La materia orgánica amorfa se presenta en mayores proporciones que en los HST (hasta 50\%). Los fitoclastos translúcidos son similares o levemente superiores a los fitoclastos opacos.

- La alteración predominante es daño mecánico y cristalización de pirita.

Los cortejos sedimentarios de bajo nivel se caracterizan por:

- La abundancia de Cheirolepidiaceae, Araucariaceae y/o esporas.

- Dentro del paleomicroplancton marino, los acritarcos adquieren mayor significación y diversidad (5 especies) en las posiciones más distales del ambiente estuarino, donde representan el grupo más abundante.

- Bajas frecuencias absolutas, con valores máximos de 45000 granos $\cdot \mathrm{g}^{-1}$.

- Los fitoclastos superan a la materia amorfa, siendo los fitoclastos translúcidos más abundantes que los fitoclastos opacos. El tipo amorfo se presenta con mayor proporción en las facies más distales.

- La alteración de los palinomorfos muestra predominio de daño mecánico.

\section{AGRADECIMIENTOS}

Los autores desean expresar su agradecimiento al Dr. Carlos Zavala, al CONICET, al Departamento de Geología y a la Secretaría General de Ciencia y Técnica (SGCyT) de la Universidad Nacional del Sur, por el apoyo brindado; así como también a los árbitros por la lectura y sugerencias recibidas oportunamente.

\section{BIBLIOGRAFÍA}

Batten, D.J. 1981. Palynofacies, organic maturation and source potential for petroleum. In: Organic maturation studies and fossil fuel exploration (Ed. J. Brooks). Academic Press, London, 201-223.

Batten, D.J. 1987. Application of palynofacies analysis to interpretation of palaeoenvironments and source potential for hydrocarbons. Notas de curso, 35 págs. (Informe inédito).

Batten, D.J. and Morrison, L. 1983. Methods of palynological preparation for palaeoenvironmentai, source potential and organic maturation studies. In: Palynology-micropalaeontology: laboratories, equipment and methods (Ed. L.I. Costa). Norwegian Petroleum Directorate, Bulletin, 2, 35-53.

Delcourt, P.A. and Delcourt, H.R. 1980. Pollen preservation and Quaternary environmental history in the southeastern United Stated. Palynology, 4, 215-231.

García, V.M., Zavala, C.A. y Quattrocchio, M.E. 1994. Relación entre análisis palinológico y análisis de facies. Aplicación al Grupo Cuyo (Jurásico medio) en la Cuenca 
Neuquina. Revista de la Asociación Geológica Argentina, 49 (1-2), 184-195.

Hall, R.L. and Westermann, G.E.G. 1980. Lower Bajocian (Jurassic) Cephalopod Faunas from Western Canada and proposed Assemblage Zones for the Lower Bajocian of North America. Paleontographica Americana, 9 (52), 93 págs.

Martínez, M.A., García, V.M. y Quattrocchio, M.E. 1996. Análisis de componentes principales aplicado al estudio palinológico del Jurásico medio de Cuenca Neuquina, Argentina. XIII Congreso Geológico Argentino y III Congreso de Exploración de Hidrocarburos, Actas, 5, 171-179.

Martínez, M.A., Quattrocchio, M.E. y Sarjeant, W.A.S. 1998. Nueva especie de Jansonia, quiste de dinoflagelado del Jurásico Medio de la Cuenca Nenquina. Su significado paleoambiental. VII Congreso Argentino de Paleontología y Bioestratigrafía, Resúmenes, pág. 24.

Menéndez, C.A. 1968. Estudio palinológico del Jurásico medio de Picún Leufú (Neuquén). Ameghiniana, Revista de la Asociación Paleontológica Argentina, Buenos Aires, 5 (10), 379-405.

Quattrocchio, M. 1980. Contribución al conocimiento de la palinología estratigráfica del Jurásico Superior en la Cuenca Neuquina. Tucumán (República Argentina). Opera Lilloana, 31, 1-59.

Quattrocchio, M.E. y Volkheimer, W. 1983. Datos palinológicos de la Formación Picún Leufú (Jurásico Superior) en su localidad tipo, Provincia de Neuquén. Parte I: Especies Marinas. Revista de la Asociación Geológica Argentina, 38 (1), 34-48.

Quattrocchio, M.E., Sarjeant, W.A.S. and Volkheimer, W. 1996a. Marine and terrestrial Jurassic microfloras of Neuquén Basin (Argentina): Palynological Zonation. In: Advances in Jurassic research (Ed. A.C. Riccardi). Transtec Publications, Switzerland. GeoResearch Forum, Vols. 1-2, 167-178.

Quattrocchio, M., Zavala, C., García, V. and Volkheimer, W. 1996b. Paleogeographic changes during the Middle Jurassic in the southern part of the Neuquén Basin, Argentina. In: Advances in Jurassic research (Ed. A.C. Riccardi). Transtec Publications, Switzerland. GeoResearch Forum, Vols. 1-2, 467-484.

Tyson, R.V. 1995. Sedimentary organic matter. Chapman \& Hall, London, 615 págs.

Volkheimer, W. 1968. Esporas y granos de polen del Jurásico del Neuquén (República Argentina). I. Descipciones sistemáticas. Ameghiniana, Revista de la Asociación Paleontológica Argentina, Buenos Aires, 5 (9), 330-370.

Volkheimer, W. 1972. Estudio palinológico de un carbón caloviano del Neuquén y consideraciones sobre los paleoclimas jurásicos en la Argentina. Revista del Museo de La Plata, 6 (Nueva Serie). Paleontología, 40, 101157.

Volkheimer, W. y Quattrocchio, M. 1975. Palinología estratigráfica del Titoniano (Formación Vaca Muerta) en el área de Caichigüe (Cuenca Neuquina). Parte A: especies terrestres. Ameghiniana, 12, 193-241.
Volkheimer, W., Caccavari, M. y Sepúlveda, E. 1977. Datos palinológicos de la Formación Ortiz (Grupo La Amarga), Cretácico inferior de la Cuenca Neuquina. Ameghiniana, 14, 59-74.

Westermann, G.E.G. y Riccardi, A.C. 1979. Middle Jurassic ammonoid fauna and biochronology of the ArgentineChilean Andes. II.- Bajocian Stephanocerataceae. Palaeontographica A, 164, 85-188.

Zavala, C.A. 1993. Estratigrafía y análisis de facies de la Formación Lajas (Jurásico medio) en el sector suroccidental de la Cuenca Neuquina. Provincia del Neuquén. República Argentina. Tesis doctoral. Departamento de Geología. UNS. 259 págs. (Informe inédito.)

Zavala, C.A. 1996a. Sequence Stratigraphy in Continental to Marine Transitions. An Example from the Middle Jurassic Cuyo Group, South Neuquén Basin, Argentina. In: Advances in Jurassic research (Ed. A.C. Riccardi). Transtec Publications, Switzerland. GeoResearch Forum, Vols. 1-2, 285-294.

Zavala, C.A. 1996b. High-Resolution Sequence Stratigraphy in the Middle Jurassic Cuyo Group, South Neuquén Basin, Argentina. In: Advances in Jurassic research (Ed. A.C. Riccardi). Transtec Publications, Switzerland. GeoResearch Forum, Vols. 1-2, 295-304.

Manuscrito recibido: 17 de julio, 1998 Manuscrito aceptado: 8 de enero, 1999

\section{ANEXO \\ LISTA TAXONÓMICA DE ESPECIES}

Se presenta a continuación la lista taxonómica de especies determinadas para el perfil Lohan Mahuida, distribuidas en los distintos grupos supragenéricos:

\section{Esporas trilete y monolete}

Alsophilidites kerguelensis Cookson, 1947

Antulsporites saevus (Balme) Archangelsky y Gamerro, 1966

Antulsporites varigranulatus (Levet-Carette) Reiser y Williams, 1969

Antulsporites sp. (Martínez, en preparación)

Asterisporites chlonovae (Döring) Venkatachala y Rawat, 1971

Baculatisporites comaumensis (Cookson) Potonié, 1956

Baculatisporites tenuis Volkheimer, 1968

Baculatisporites cf. tenuis Volkheimer, 1968

Baculatisporites spp.

Biretisporites sp. (en Volkheimer y Quattrocchio, 1975)

Biretisporites spp.

Clavatisporites bagualensis (Volkheimer) (Martínez, en prep. )

Clavatisporites spp. 
Concavisporites laticrassus Volkheimer, 1972

Deltoidospora australis (Couper) Pocock, 1970

Deltoidospora minor (Couper) Pocock, 1970

Deltoidospora neddeni Pflug, 1953

Deltoidospora spp.

Dictyophyllidites mortoni (De Jersey, 1959) Playford y Dettmann, 1965

Dictyophyllidites spp.

Duplexisporites spp.

Espora tipo 1 (Martínez, en prep.)

Foveotriletes microfoveolatus Menéndez, 1968

Gleicheniidites argentinus Volkheimer, 1972

Gleicheniidites sp. (García, en prep.)

Granulatisporites sp. A (en Volkheimer, 1968)

Granulatisporites sp. D (en Volkheimer, 1972)

Granulatisporites spp.

Interulobites variabilis Volkheimer y Quattrocchio, 1975

Ischyosporites marburgensis De Jersey, 1963

Ischyosporites variegatus (Couper, 1958) Schulz, 1967

Ischyosporites spp.

Klukisporites labiatus (Volkheimer, 1968) Baldoni y Archangelsky, 1983

Klukisporites spp.

Leptolepidites major Couper, 1958

Leptolepidites spp.

Neoraistrickia cf. truncata (Cookson) Potonié, 1956 (Martínez, en prep.)

Neoraistrickia truncata (Cookson) Potonié, 1956

Osmundacidites araucanus Volkheimer, 1972

Osmundacidites spp.

Pilosisporites sp. (Martínez, en prep.)

Polycingulatisporites spp.

Punctatosporites scabratus (Couper) Norris, 1965

Retitriletes austroclavatidites (Cookson) Döring, Krutzsch, Mai y Schulz, 1963

Retitriletes spp.

Rugulatisporites neuquenensis Volkheimer, 1972

Todisporites minor Couper, 1958

Trilites densiverrucosus Menéndez, 1968

Trilobosporites (?) spp.

Uvaesporites minimus Volkheimer, 1968

Verrucosisporites varians Volkheimer, 1972

Verrucosisporites cf. opimus Manum, 1962 (en Volkheimer, 1968)

cf. Verrucosisporites opimus Manum,1962 (Martínez, en prep.)

Verrucosisporites cf. walloonensis De Jersey, 1959 (en Volkheimer, 1968)

Verrucosisporites spp.

\section{Caytoniaceae}

Vitreisporites pallidus (Reissinger) Nilsson, 1958

Vitreisporites sp. (en Volkheimer, 1968)

Vitreisporites spp.

\section{Podocarpaceae}

Alisporites robustus Nilsson, 1958

Alisporites sp. A (en Volkheimer, 1968)

Alisporites spp.

Callialasporites dampieri (Balme) Dev, 1961

Callialasporites microvelatus Schulz, 1966

Callialasporites segmentatus (Balme) Srivastava, 1963

Callialasporites trilobatus (Balme) Dev, 1961

Callialasporites spp.

Dacrycarpites spp.

Microcachryidites spp.

Phrixipollenites spp.

Podocarpidites cf. ellipticus Cookson, 1947 (en Volkheimer, 1968)

Podocarpidites cf. otagoensis Couper, 1953 (en Volkheimer, 1968)

Podocarpidites verrucosus Volkheimer, 1972

Podocarpidites spp.

Podosporites (?) spp.

\section{Araucariaceae}

Araucariacites australis Cookson, 1947

Araucariacites fissus Reiser y Williams, 1969

Araucariacites pergranulatus Volkheimer, 1968

Araucariacites spp.

Inaperturopollenites indicus Srivastava, 1966

Inaperturopollenites microgranulatus Volkheimer, 1972

Inaperturopollenites turbatus Balme, 1957

Inaperturopollenites sp. A (en Volkheimer, 1972)

Inaperturopollenites sp. B (en Volkheimer, 1972)

Inaperturopollenites spp.

\section{Cheirolepidiaceae}

Classopollis simplex (Danzé, Corsin y Laveine) Reisser y Williams, 1969

Classopollis cf. classoides (Pflug, 1953) Pocock y Jansonius, 1961 (en Volkheimer, 1968)

Classopollis major Groot y Groot, 1962

Classopollis cf. torosus (Reissinger, 1950; Couper, 1958) emend. Burger, 1965 (en Volkheimer, 1968)

Classopollis spp.

Gliscopollis tersus Norris, 1965

\section{Cycadales/Bennettitales}

Cycadopites adjectus (De Jersey, 1962) Volkheimer y Quattrocchio, 1975 
Cycadopites granulatus (De Jersey, 1962) De Jersey, 1964 Cycadopites nitidus (Balme 1957) De Jersey, 1964

Cycadopites punctatus Volkheimer, 1968

Cycadopites spp.

Monosulcites carpentieri Delcourt y Sprumont, 1955

Monosulcites aff. minimus Cookson, 1947 (en Menéndez, 1968)

Monosulcites sp. A (en Volkheimer, 1972)

Monosulcites sp. B (en Volkheimer y Quattrocchio, 1975)

Monosulcites sp. (en Quattrocchio, 1980)

Monosulcites spp.

\section{Chlorococcales}

Botryococcus spp.

\section{Acritarcos}

Baltisphaeridium debilispinum Wall y Downie, 1963

Baltisphaeridium sp. (García, en prep.)

Comasphaeridium sp. (García, en prep.)

Micrhystridium fragile Deflandre, 1947

Micrhystridium cf. rarispinum Sarjeant, 1960 (Martínez, en prep.)

Micrhystridium sp. (García, en prep.)

Micrhystridium spp.

Solisphaeridium sp. (García, en prep.)
Solisphaeridium stimuliferum (Deflandre, 1938) Staplin, Jansonius y Pocock, 1965

\section{Dinoquistes}

Acanthaulax (?) downiei (Sarjeant, 1960) Sarjeant, 1976

Acanthaulax spp.

Endoscrinium spp.

Escharisphaeridia pocockii (Sarjeant, 1968) Erkmen y Sarjeant, 1980

Escharisphaeridia spp.

Jansonia sp (Martínez, Quattrocchio y Sarjeant, en prep.)

Kalyptea cf. jurassica Alberti, 1961 (Martínez, en prep.)

Nannoceratopsis gracilis (Alberti ,1961) Evitt, 1962

Nannoceratopsis sp. (García, en prep.)

\section{Prasinophyceae}

Cymatiosphaera eupeplos (Valensi, 1948) Deflandre, 1954

Cymatiosphaera sp. (García, en prep.)

Leiosphaeridia sp. C (en Quattrocchio y Volkheimer, 1983)

Leiosphaeridia cf. L. sp. B (en Volkheimer et al., 1977)

Leiosphaeridia cf. hyalina (Deflandre, 1941) Downie, 1957 (en Quattrocchio, 1980)

Leiosphaeridia spp.

Pleurozonaria picunensis Quattrocchio, 1980

Pterosphaeridia spp. 